\title{
Double point-homogeneous spherical curves
}

\author{
Guy Valette
}

\begin{abstract}
A curve is, in this paper, the image of the circle $S^{1}$ under an immersion $f$ into $S^{2}, \mathbb{R}^{2}$ or the real projective plane $P_{2}(\mathbb{R})$, such that every multiple point of $f$ is an ordinary double point. Such a curve $C$ is double pointhomogeneous or DP-homogeneous when the group of diffeomorphisms (of $S^{2}, \mathbb{R}^{2}$ or $P_{2}(\mathbb{R})$ ) preserving $C$ has a transitive action on the set of its double points. The orbits of DP-homogeneous curves in $S^{2}$ are totally determined; using combinatorial methods, we prove that they fall into five countably infinite families ; the description of every family is illustrated by drawings of some representatives with a small number of double points. As a corollary, we obtain a similar classification of the DP-homogeneous curves in $\mathbb{R}^{2}$. We also propose a conjecture about the classification of DP-homogeneous curves in $P_{2}(\mathbb{R})$.
\end{abstract}

\section{Introduction}

The curves considered in this paper are generic which means that each one is the image of an immersion $f$ of the circle $S^{1}$ into a two-dimensional manifold $M$ such that every multiple point of $f$ is an ordinary double point. Such a curve $C$ is said to be double point-homogeneous or DP-homogeneous if, for every pair $(p, q)$ of double points of $C$, there is a diffeomorphism of $M$ which preserves $C$ and sends $p$ onto $q$. The main result of this paper is the classification of the orbits of DP-homogeneous spherical curves (case $M=S^{2}$ ) under the action of the group of all diffeomorphisms of $S^{2}$. A consequence of this is the classification of DP-homogeneous plane curves (case $M=\mathbb{R}^{2}$ ) under the action of the group of

Received by the editors in November 2015.

Communicated by J. Doyen.

2010 Mathematics Subject Classification : Primary 53A04; Secondary 52C20, 58D19.

Key words and phrases : smooth closed curve, double point, transitive action, tiling. 
all diffeomorphisms of $\mathbb{R}^{2}$. We sometimes say that two curves are equivalent if they belong to the same orbit.

Examples of DP-homogeneous plane curves are presented in Figures 1 and 2. Any two different curves among the eight shown there are not equivalent. But if we denote by $C$ a curve in Figure 1 and by $D$ the curve in Figure 2 having the same number of double points as $C$, and if we map $\mathbb{R}^{2}$ onto open subsets of $S^{2}$ by diffeomorphisms $F$ and $G$, then the spherical curves $F(C)$ and $G(D)$ are equivalent. This remark suggests the existence of a first infinite family of orbits of DP-homogeneous spherical curves, the family $\mathbf{P}$, with representatives of $\mathbf{P}_{1}$ to $\mathbf{P}_{4}$ shown in Figure 3.
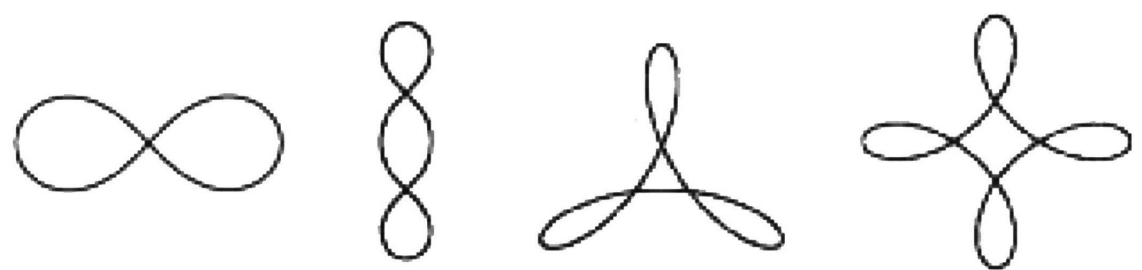

FIGURE 1: Four DP-homogeneous plane curves which are not equivalent with respect to diffeomorphisms of $\mathbb{R}^{2}$. They belong to Family $\mathbf{P}^{\prime}$ (see Section 4 ).
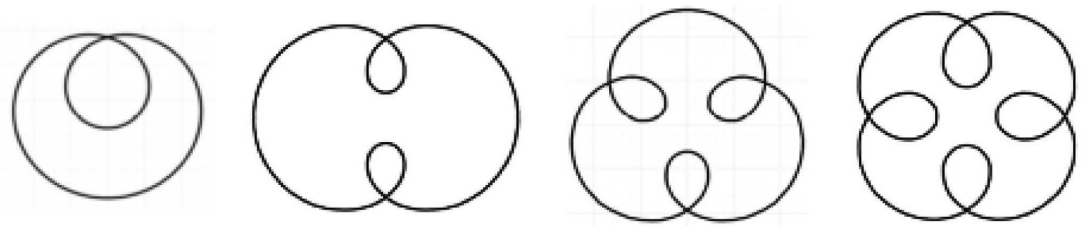

FIGURE 2: Four DP-homogeneous plane curves which are not equivalent to those of Figure 1. They belong to Family $\mathbf{P}^{\prime \prime}$ (see Section 4).
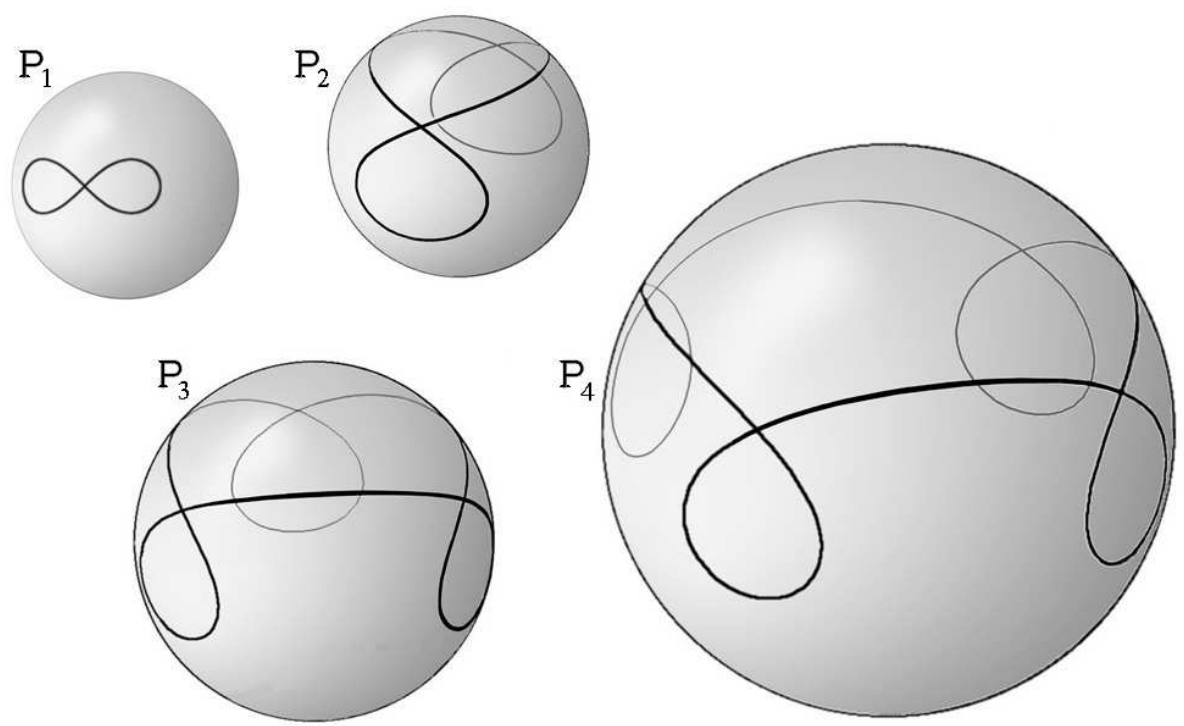

Figure 3: Representatives of the first four elements of the family $\mathbf{P}$ of orbits of DPhomogeneous spherical curves. 
We shall prove that the other orbits of DP-homogeneous spherical curves are classified in a natural way into four families presented in Figures 4, 5, 6 and 7 by means of representatives.
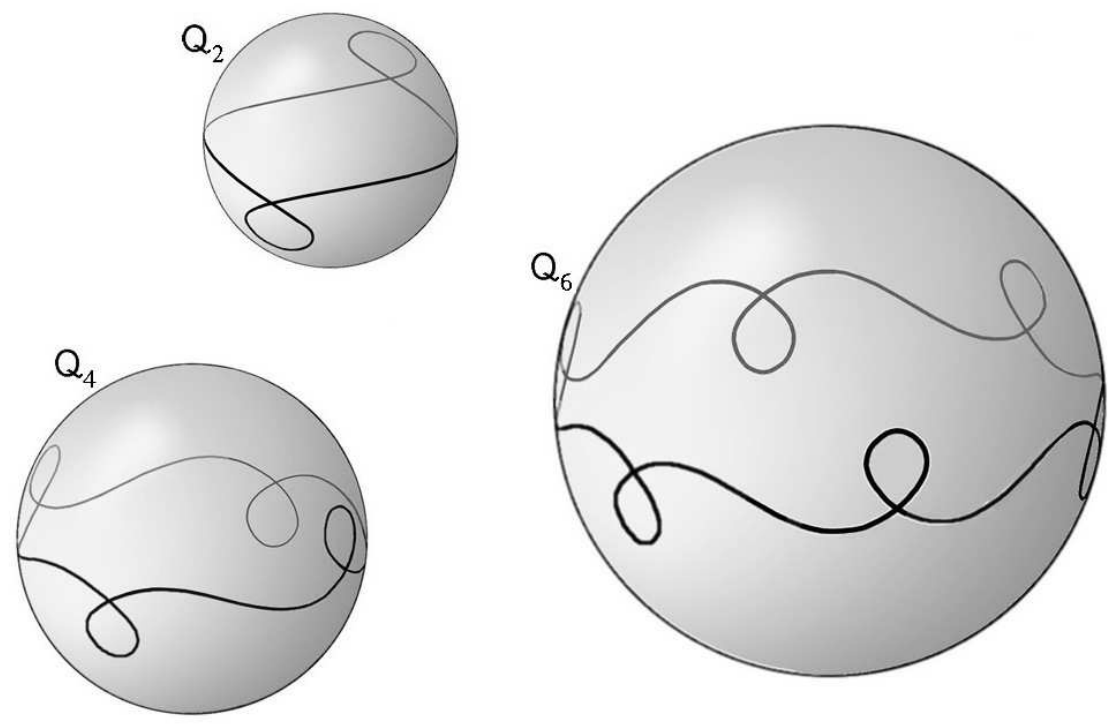

FigURE 4: Representatives of the first three elements of the family $\mathbf{Q}$.
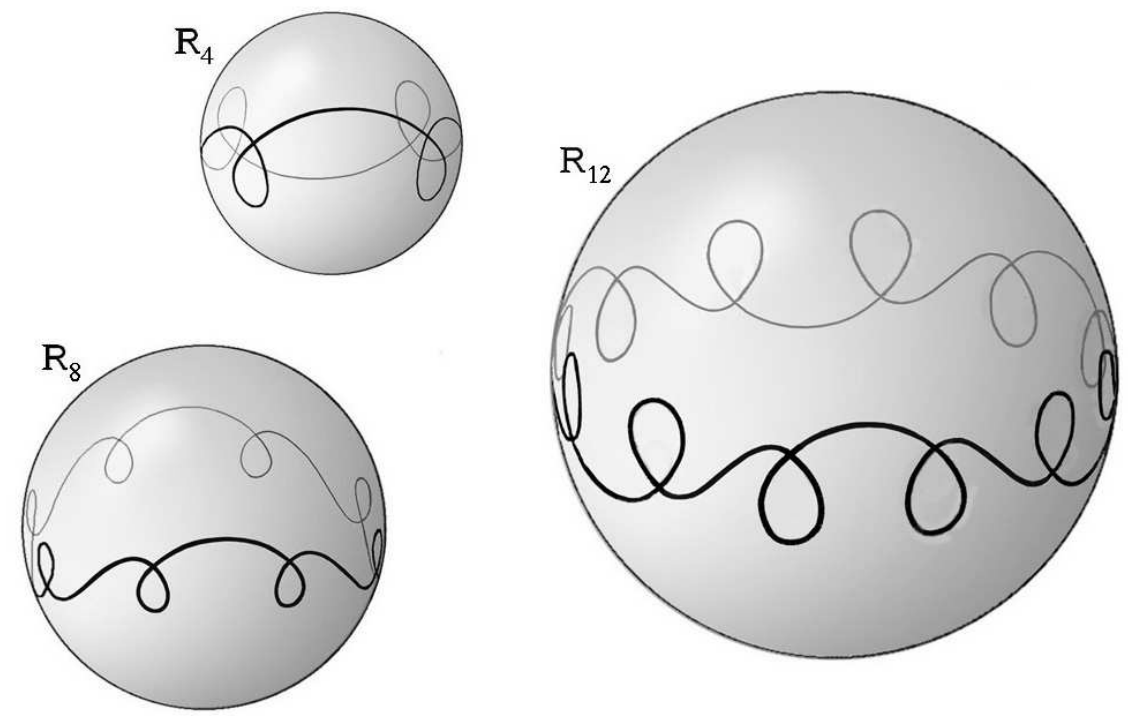

Figure 5: Representatives of the first three elements of the family $\mathbf{R}$. 

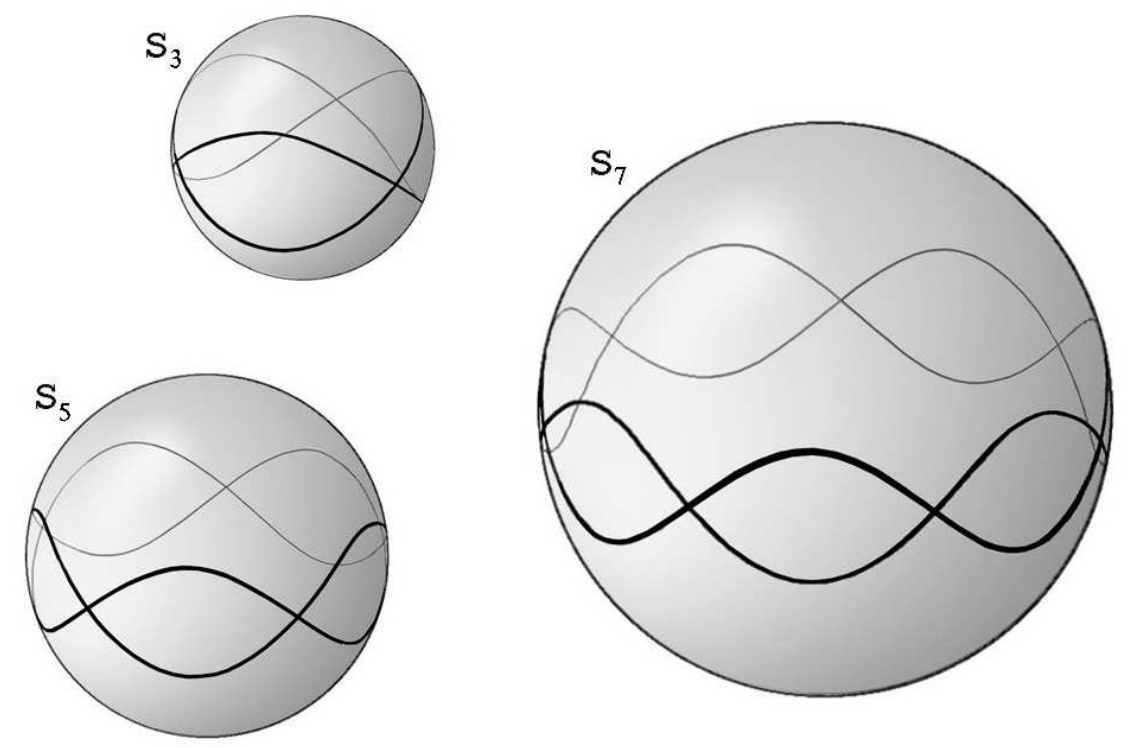

FigURE 6: Representatives of the first three elements of the family $\mathbf{S}$.
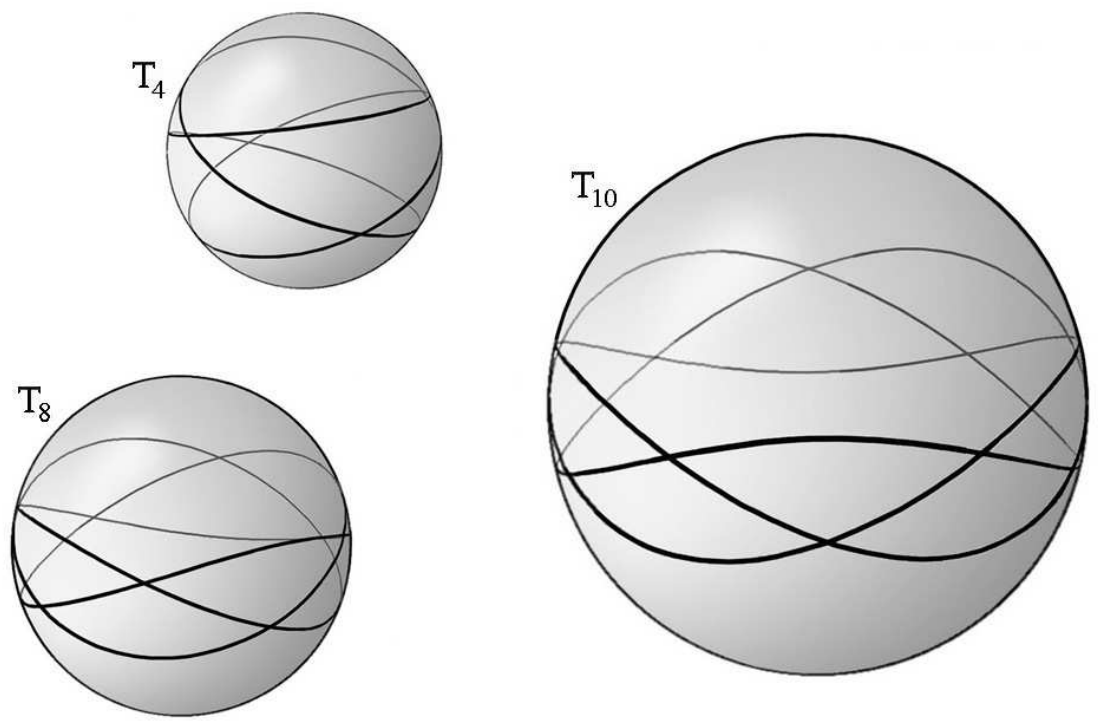

FIGURE 7: Representatives of the first three elements of the family $\mathbf{T}$.

Let us denote by $O(n)$ the number of orbits of DP-homogeneous spherical curves having exactly $n$ double points $(n \geq 1)$; a first consequence of our classification is the fact that the function $n \rightarrow O(n)$ is completely known: its first fourteen values are $1,2,2,4,2,2,2,4,2,3,2,3,2,3$, and the next values satisfy the recurrence $O(n)=O(n-12)$.

Another consequence of our classification is the analogous classification of the orbits of DP-homogeneous plane curves. Two infinite families were already presented in Figures 1 and 2. There is a third one: representatives of its first three elements are shown in Figure 8. 

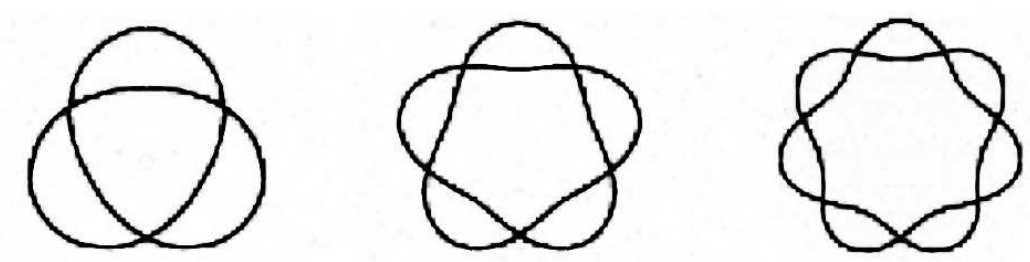

FIGURE 8: Representatives of the first elements of the third family of orbits of DPhomogeneous plane curves. They belong to Family $\mathbf{S}^{\prime}$ (see Section 4).

\section{Statement of the main result}

The following definitions, where $M$ denotes $\mathbb{R}^{2}$ or $S^{2}$, are useful for the description of DP-homogeneous (plane or spherical) curves.

DEFINITIONS: A curvilinear m-gon $(m \geq 1)$ is any subset $D$ of $M$ which is homeomorphic to a closed disk and whose boundary $B$ is a closed curve which is smooth everywhere excepted in $m$ angular points, called vertices. If $m>1$, a side of $D$ is an arc of $B$ joining neighboring vertices; if $m=1$, it is $B$.

A vertex $a$ of the curvilinear $m$-gon $D$ is said to be salient if the measure of the interior angle of $D$ in $a$ is smaller than $\pi$, and is re-entrant if this measure is greater than $\pi$.

Let $C$ be a curve having $n$ double points $(n \geq 1)$; a curvilinear $m$-gon is said to be inscribed in $C$ if its sides are arcs of $C$ joining neighboring double points if $m>1$, the same double point if $m=1$. An example of an inscribed 5-gon is given in Figure 9.

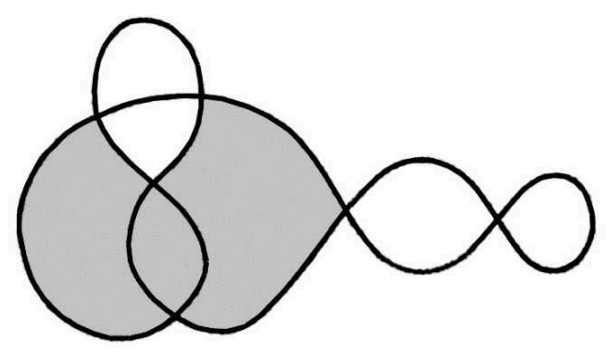

FIGURE 9: A 5-gon (coloured in grey) inscribed in a curve.

A curve $C$ in $M$ determines a tiling of $M$, whose tiles are the closures of the connected components of $M \backslash C$; for brevity's sake, we will say that the tiles of this tiling are the tiles of $C$. Such a tile is biangular (resp. triangular) if it is a curvilinear 2-gon (resp. 3-gon) with salient vertices. 
THEOREM: If a DP-homogeneous spherical curve has at least one double point, then (under the group of all diffeomorphisms of $S^{2}$ ) it belongs to one orbit of one of the following five families:

1) The family $\mathbf{P}$ is the sequence of orbits $\mathbf{P}_{1}, \mathbf{P}_{2}, \mathbf{P}_{3}, \ldots, \mathbf{P}_{n}, \ldots$ where any element of $\mathbf{P}_{1}$ is a figure-eight curve and, if $n>1$, any element $C$ of $\mathbf{P}_{n}$ is a curve (with $n$ double points) one tile of which is a curvilinear n-gon with salient vertices, each of these vertices being also the vertex of a curvilinear 1-gon inscribed in $C$. Examples of elements of $\mathbf{P}_{1}$, $\mathbf{P}_{2}, \mathbf{P}_{3}$ and $\mathbf{P}_{4}$ are shown on the four spheres of Figure 3.

2) The family $\mathbf{Q}$ is the sequence of orbits $\mathbf{Q}_{2}, \mathbf{Q}_{4}, \mathbf{Q}_{6}, \ldots, \mathbf{Q}_{2 m}, \ldots$ where any element $C$ of $\mathbf{Q}_{2 m}$ is a curve (with $2 m$ double points) in which a curvilinear $2 m$-gon $D$ is inscribed; the vertices of $D$ are alternately salient and re-entrant and each of them is also the vertex of a curvilinear 1-gon inscribed in C. Examples of elements of $\mathbf{Q}_{2}, \mathbf{Q}_{4}$ and $\mathbf{Q}_{6}$ are shown on the three spheres of Figure 4 .

3) The family $\mathbf{R}$ is the sequence of orbits $\mathbf{R}_{4}, \mathbf{R}_{8}, \mathbf{R}_{12}, \ldots, \mathbf{R}_{4 m}, \ldots$ where any element C of $\mathbf{R}_{4 m}$ is a curve (with $4 m$ double points) in which a curvilinear $4 m$-gon $D$ is inscribed; every vertex of $D$ has one salient neighbour and one re-entrant neighbour, and is also the vertex of a curvilinear 1-gon incribed in C. Examples of elements of $\mathbf{R}_{4}, \mathbf{R}_{8}$ and $\mathbf{R}_{12}$ are shown on the spheres of Figure 5.

4) The family $\mathbf{S}$ is the sequence of orbits $\mathbf{S}_{3}, \mathbf{S}_{5}, \mathbf{S}_{7}, \ldots, \mathbf{S}_{2 m+1}, \ldots$ where any element $C$ of $\mathbf{S}_{2 m+1}$ is a curve (with $2 m+1$ double points) in which two curvilinear $(2 m+1)$-gons with the same salient vertices are inscribed; they are separated by a chain of $2 m+1$ biangular tiles with salient vertices. Examples of elements of $\mathbf{S}_{3}, \mathbf{S}_{5}$ and $\mathbf{S}_{7}$ are shown on the three spheres of Figure 6.

5) The family $\mathbf{T}$ is the sequence of orbits $\mathbf{T}_{4}, \mathbf{T}_{8}, \mathbf{T}_{10}, \ldots, \mathbf{T}_{6 m-2}, \mathbf{T}_{6 m+2}, \ldots$ where any element $C$ of $\mathbf{T}_{6 m \pm 2}$ is a curve (with $6 m \pm 2$ double points) whose two tiles are curvilinear $(3 m \pm 1)$-gons with salient vertices; they are strictly separated by a belt of $6 m \pm 2$ triangular tiles. If $6 m \pm 2>4$, then the tiling of $C$ is combinatorially equivalent to the natural tiling of the boundary of an antiprism whose bases are $(3 m \pm 1)$-gons. Examples of elements of $\mathbf{T}_{4}, \mathbf{T}_{8}$ and $\mathbf{T}_{10}$ are shown on the spheres of Figure 7.

\section{Gauss diagrams and proofs}

Our proof of the Theorem uses diagrams introduced by Gauss [Ga]. We define them via codes of curves which are similar to the Gauss codes used in knot theory.

NOTATIONS AND DEFINITIONS: Let $C=f\left(S^{1}\right)$ be a spherical curve with $n$ double points $(n>0)$. In order to define a Gauss code of $C$, we first give a name (letter with or without subscript) to each double point of $C$ and then write their names following the order in which $f(u)$ meets them when $u$ runs along $S^{1}$; the word (of length $2 n$ ) so obtained is a Gauss code of $C$, which is defined (after the choice of names) up to an element of the dihedral group $D_{2 n}$.

Every Gauss code $\Omega$ of $2 n$ letters may be represented by a Gauss diagram of $\operatorname{order} n$, i.e. a plane figure $\Gamma$ consisting of 
(i) a circle $\gamma$ of the Euclidean plane,

(ii) the vertices of a regular $2 n$-gon $P$ inscribed in $\gamma$, also called vertices of $\Gamma$, denoted by the letters of $\Omega$ in such a way that neighboring vertices of $P$ correspond to successive letters of $\Omega$,

(iii) the $n$ chords joining the vertices which have the same name.

Figure 10 describes an example of this representation.
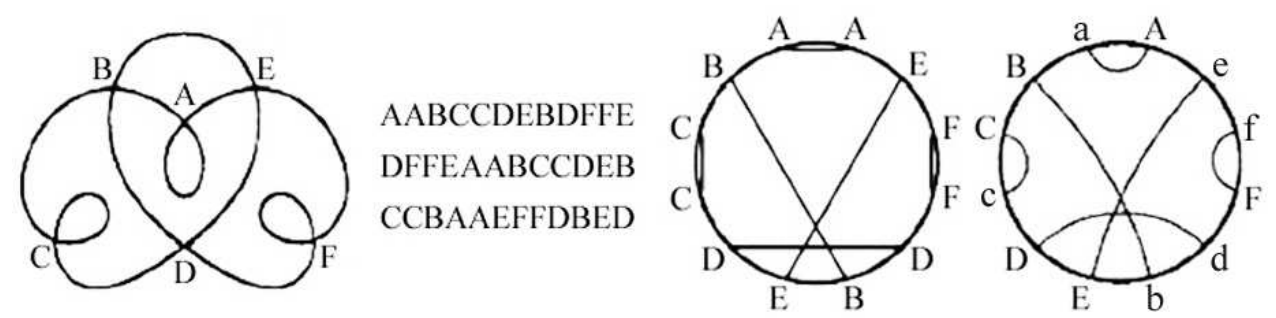

FIGURE 10: From left to right : a curve whose double points are A, B, C, D, E, F; three equivalent Gauss codes of this curve ; a Gauss diagram $\Gamma$ of these codes and of the curve ; a variant to $\Gamma$ with a better visibility (it is sometimes useful to take different but similar names for the endpoints of a chord).

Let $K$ be a chord of the Gauss diagram $\Gamma$; the step of $K$ is the minimum number of sides of $P$ needed to join the endpoints of $K$ along the boundary of $P$. An $s$-chord is a chord whose step is $s$ (in the example of Fig. 10, $\Gamma$ has three 1-chords, one 3-chord and two 5-chords).

One easily proves that every chord in the Gauss diagram of any plane or spherical curve has an odd step and that if a curve is DP-homogeneous, then all its chords have the same step (note that the converse is not true: for example, there is a spherical curve with three double points which is not DP-homogeneous, but whose Gauss diagram has only 1-chords).

LEMMA 1: If a spherical curve $C$ with $n$ double points is DP-homogeneous, then its Gauss diagram $\Gamma$ is invariant under the group $C_{n}$ of rotations whose angles are multiples of $2 \pi / n$.

Proof: Let $s$ be the common step of the chords of $\Gamma$ and let [0], [1], ... , [2n-1] be the vertices of the polygon $\mathrm{P}$ used in the definition of $\Gamma$ (the sides of $\mathrm{P}$ are the segments $[[j],[j+1]]$, addition being done mod $2 n)$. As the Lemma is obvious when $s=1$ or $s=n$, we may assume that $1<s<n$ and prove the assertion by contradiction. Let us agree that two chords are neighboring if one of them is the image of the other by a rotation of $\pi / n$.

If the assertion were false, then we could find in $\Gamma$ two neighboring chords, one of them being $[[a],[a+s]]$ and the other $[[a+1],[a+1+s]]$; two possibilities occur: either one of these chords is neighboring with a third chord, or not.

$\alpha$ ) The first assumption implies that a double point of $C$ is a vertex of two biangular tiles of $C$; as $C$ is DP-homogeneous, all the double points of $C$ have the same property; this implies that, for every vertex $[j]$, the segment $[[j],[j+s]]$ is a chord of $\Gamma$, which is only possible when $s=n$, contradicting the condition $1<s<n$.

$\beta$ ) The second assumption and the DP-homogeneity imply that the set of chords 
of $\Gamma$ can be partitioned into disjoint pairs of neighboring chords and consequently, that the set of vertices of $\Gamma$ can be partitioned into disjoint pairs of neighboring vertices which are endpoints of neighboring chords. Since $[[a],[a+s]]$ and $[[a+1],[a+1+s]]$ are such chords, the number of vertices of $\Gamma$ between $[a+1]$ and $[a+s]$ is equal to $s-2$, an odd number, giving the contradiction.

The notation $\Gamma(n, s)$ will be used for any plane diagram consisting of

(i) a circle $\gamma$ of the Euclidean plane,

(ii) the vertices of a regular $2 n$-gon $P$ inscribed in $\gamma$,

(iii) $n$ chords of $\gamma$ with odd step s joining pairs of vertices of $P$, whose union is invariant under the rotation group $C_{n}$.

Note that, given integers $n$ and $s$ with $s$ odd and $1 \leq s \leq n$, there is essentially one diagram with these properties. Examples are drawn in Fig. 11.
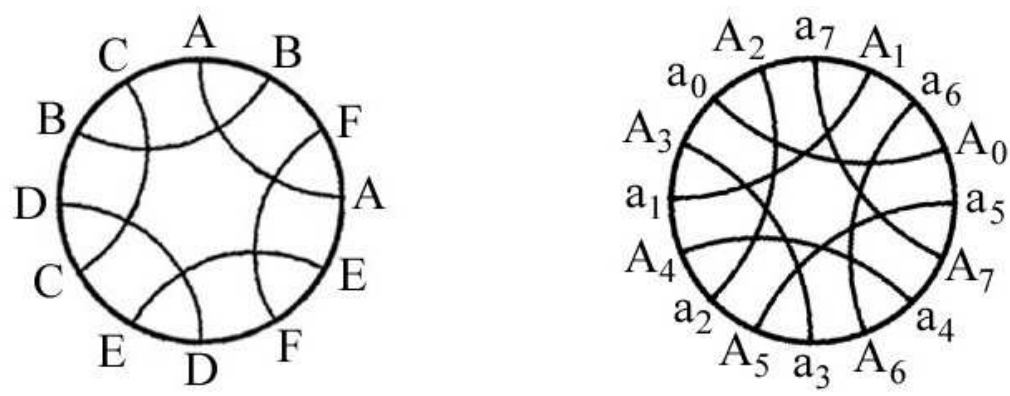

FIGURE 11: The diagrams $\Gamma(6,3)$ and $\Gamma(8,5) ; A F B A C B D C E D F E$ may be a code for $\Gamma(6,3)$, and $A_{0} a_{6} A_{1} a_{7} A_{2} a_{0} A_{3} a_{1} A_{4} a_{2} A_{5} a_{3} A_{6} a_{4} A_{7} a_{5}$ for $\Gamma(8,5)$.

In the proof of Lemma 2, we use a procedure found by L. Lovasz and M.L. Marx [LM] to decide whether or not a word of $2 n$ characters ( $n$ symbols occurring twice) is the Gauss code of a spherical curve. In order to increase the readability of our paper, we now recall three definitions and two properties given in [LM].

DEFINITIONS: If a word has the form $A \alpha A \beta$ where $\alpha$ and $\beta$ are non-empty sequences, then the vertex split at $A$ is the change from this word to $\alpha^{-1} \beta$ where $\alpha^{-1}$ has the same letters as $\alpha$ but in the opposite order.

The loop removal at $A$ of the word $A \alpha A \beta$ is the change from this word to the one obtained from $\beta$ by deleting all the letters which occur in $\alpha$.

A reduced word of a word $\Omega$ is a non-empty word obtained from $\Omega$ after a finite number of changes (vertex splits or loop removals).

PROPERTY 1 ("biparity condition" in [LM]): If the Gauss code of a spherical curve with at least two double points $A$ and $B$ has the form $A \alpha A \mu B \beta B \gamma$ where $\alpha$, $\mu, \beta, \gamma$ are finite (possibly empty) sequences of letters, then $\alpha$ and $\beta$ have an even number of common letters.

PROPERTY 2 ("Theorem" in [LM]): A word $\Omega$ wherein each letter occurs twice is a Gauss code of a spherical curve if and only if no reduced word of $\Omega$ has the form $A_{1} A_{2} \ldots A_{m} A_{1} A_{2} \ldots A_{m}$ with $m$ even.

LEMMA 2: If C is a DP-homogeneous spherical curve, then its Gauss diagram belongs to one of the three families described below and shown in Fig. 12:

a) the family $\mathbf{A}$ consists of diagrams $\Gamma(n, 1)$ where $n \in \mathbb{N}_{0}$, 
b) the family $\mathbf{B}$ consists of diagrams $\Gamma(n, n)$ where $n=2 m+1\left(m \in \mathbb{N}_{0}\right)$,

c) the family $\mathbf{C}$ consists of diagrams $\Gamma(n, s)$ where $n$ and $s$ depend on $m \in \mathbb{N}_{0}$ in one of the following ways:

either $n=6 m-2$ and $s=4 m-1 \quad$ or $\quad n=6 m+2$ and $s=4 m+1$.

A
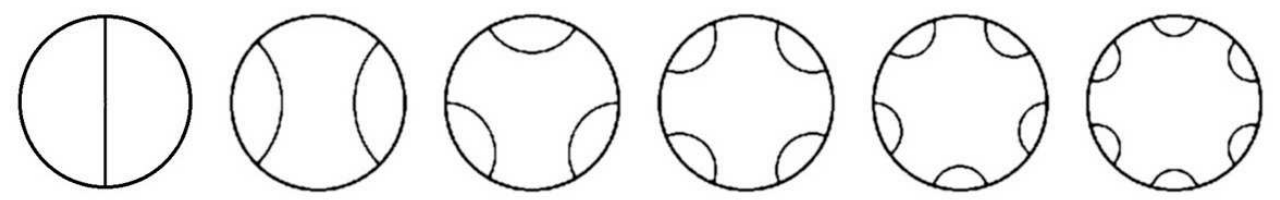

etc.

B
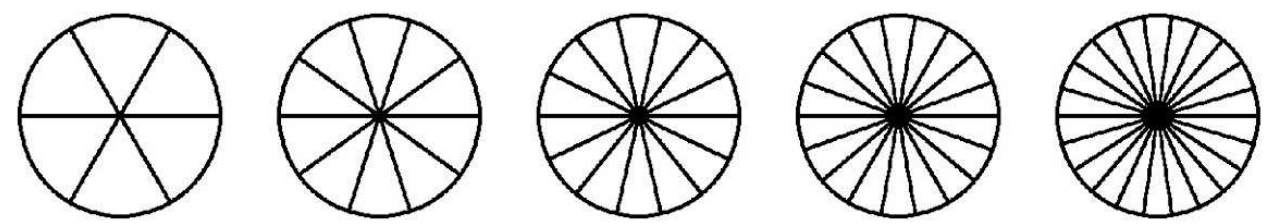

etc.

C
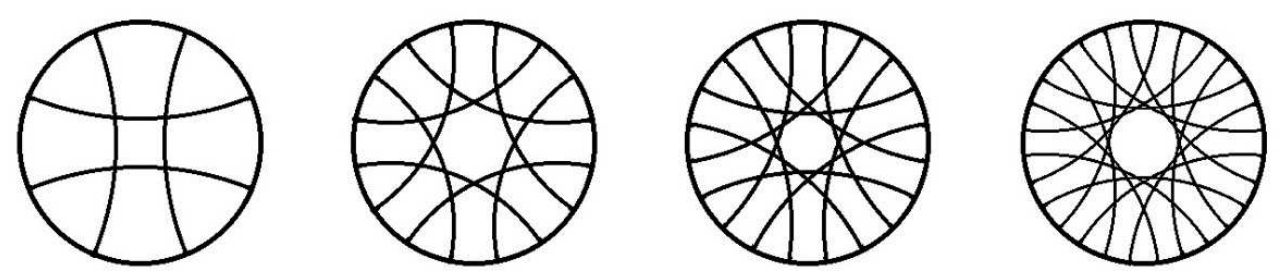

etc.

FIGURE 12: The three families of diagrams considered in Lemma 2.

Proof: The family A (resp. B) consists of all the diagrams described in Lemma 1 when the step $s$ equals 1 (resp. $n$ ). Hence it remains to exclude, among the diagrams $\Gamma(n, s)$ such that $2<s<n$, those which are neither of the form $n=6 m-2$ and $s=4 m-1$ nor of the form $n=6 m+2$ and $s=4 m+1$. In other words, we must exclude all diagrams $\Gamma(n, s)$ for which $n$ is odd and, among the diagrams with $n$ even, those for which $2 n$ does not belong to $\{3 s-1,3 s+1\}$. Suppose on the contrary that there is a spherical curve $C$ whose Gauss diagram must be excluded. By Lemma 1, its Gauss code $\Omega$ may be written as $A \alpha A \mu B \beta B \gamma$ where the words $\mu$ and $\gamma$ are possibly empty and $|\alpha|=|\beta|=s-1$. $(|\sigma|$ denotes the number of letters of the word $\sigma$ ). We distinguish three cases in order to get a contradiction.

a) $2 n$ is greater than $3 s+1$. If $s \equiv 3 \bmod 4$, then we take $\mu=\varnothing$ in the notation above for $\Omega$; if $s \equiv 1 \bmod 4$, then we choose $|\mu|=2$; in both cases, the number of common letters of $\alpha$ and $\beta$ is odd, contradicting Property 1 .

$\beta) n$ is odd and $2 n \leq 3 s+1$. Since $|\alpha|=|\beta|=s-1$, we have

$$
\overline{|\mu|+|\gamma|=2 n-2(s-1)}-4=2 n-2 s-2 \leq 3 s+1-2 s-2=s-1 \text {, }
$$

which shows that a letter cannot appear twice in any word $\alpha, \mu, \beta$ or $\gamma$; moreover, the distance between any letter of $\mu$ and any letter of $\gamma$ is at least $s+2$; this implies that any letter of $\mu \cup \gamma$ appears also in $\alpha \cup \beta$. Hence the number of letters with two occurences in $\alpha \cup \beta$ is equal to $(2(s-1)-(2 n-2 s-2)) / 2$ i.e. $2 s-n$, which implies that the number of letters common to $\alpha$ and $\beta$ is the odd number $2 s-n$, contradicting Property 1.

$\gamma) n$ is even and $2 n<3 s+1$. We use the notation introduced in the second example of Fig.11 for the Gauss code of a curve $C$ with diagram $\Gamma(n, s)$ : so 
$A_{k}=[2 k]$ and $a_{k}=[2 k+s]$ if we identify the set of vertices of $\Gamma(n, s)$ with $\mathbb{Z} /(2 n)$. By Lemma 1, a Gauss code for $\Gamma(6,5)$ can be written as

$$
\Omega(6,5)=A_{0} a_{4} A_{1} a_{5} A_{2} a_{0} A_{3} a_{1} A_{4} a_{2} A_{5} a_{3} ;
$$

if $\Omega(6,5)$ were the Gauss code of a spherical curve, then a vertex split of $\Omega(6,5)$ at $A_{0}$ would produce the Gauss code $\Omega^{\prime}$ of a spherical curve, but this is not so because $\Omega^{\prime}$ does not satisfy the biparity condition, a contradiction. In the case $n>7$, a Gauss code for $\Gamma(n, s)$ may be written as

$$
\Omega(n, s)=A_{0} a_{g} A_{1} a_{g+1} A_{2} \ldots a_{n-1} A_{h} a_{0} A_{h+1} a_{1} \ldots A_{g} a_{g-h} A_{g+1} a_{g-h+1} \ldots A_{n-1} a_{g-1}
$$

where $g=(2 n-s+1) / 2$ and $h=(s-1) / 2$. In this case two changes are needed to conclude: the first one is the vertex split of $\Omega(n, s)$ at $A_{0}$, giving the word

$$
A_{h} a_{n-1} A_{h-1} \ldots A_{2} a_{g+1} A_{1} a_{g} A_{h+1} a_{1} \ldots A_{g} a_{g-h} A_{g+1} a_{g-h+1} \ldots A_{n-1} a_{g-1}
$$

or the equivalent word

$$
\Omega^{\prime}=A_{g+1} a_{g-h+1} \ldots A_{n-1} a_{g-1} A_{h} a_{n-1} A_{h-1} \ldots A_{2} a_{g+1} A_{1} a_{g} A_{h+1} a_{1} \ldots A_{g} a_{g-h}
$$

also written $\Omega^{\prime}=A_{g+1} \alpha a_{g+1} \beta$ if we set

$$
\alpha=a_{g-h+1} \ldots A_{n-1} a_{g-1} A_{h} a_{n-1} A_{h-1} \ldots A_{2} \text { and } \beta=A_{1} a_{g} A_{h+1} a_{1} \ldots A_{g} a_{g-h}
$$

Finally, a loop removal of $\Omega^{\prime}$ at $A_{g+1}$ creates the reduced word $A_{1} a_{g} a_{1} A_{g}$ which means, according to Property 2 , that $\Omega(n, s)$ is not the Gauss code of a spherical curve, contrary to the assumption.

Proof of the Theorem: Every curve described in the Theorem is clearly DP-homogeneous; moreover, if it belongs to one of the families $\mathbf{P}, \mathbf{Q}$ or $\mathbf{R}$, then its Gauss diagram belongs to family $\mathbf{A}$ while, if it belongs to family $\mathbf{S}$ (resp. $\mathbf{T}$ ), then its Gauss diagram belongs to family B (resp. C). So its remains to show that every DP-homogeneous spherical curve $C$ with $n$ double points belongs to one of the families $\mathbf{P}, \mathbf{Q}, \mathbf{R}, \mathbf{S}$ or $\mathbf{T}$. The rest of the proof has three parts, corresponding to the three possible families $\mathbf{A}, \mathbf{B}$ and $\mathbf{C}$.

a) Curves with diagram in family $\mathbf{A}$. If the Gauss diagram of $C$ is $\Gamma(1,1)$, then $C$ is clearly a figure-eight curve and all such curves form the orbit $\mathbf{A}_{1}$. If the Gauss diagram of $C$ is $\Gamma(n, 1)$ with $n>1$, then $C$ is a union of $n$ loops and $n$ arcs connecting neighboring double points; these arcs form a Jordan curve $B$, which is boundary of two curvilinear $n$-gons.

$\alpha$ ) If the loops of $C$ at a double point and at its two neighbors (only one if $n=2$ ) are on the same side of $B$, then this property is true for every double point, and so $C$ belongs to the orbit $\mathbf{P}_{n}$.

$\beta$ ) If the loop of $C$ at a double point is on one side of $B$ while the loops at its neighbors are on the other side, then this property is true for every double point, which implies that $n$ is even and that $C$ belongs to the orbit $\mathbf{Q}_{n}$.

$\gamma$ ) If the loops of $C$ at the neighbors of a double point are not on the same side of $B$, then this property is true for every double point, and so $n$ is a multiple of 4 and $C$ belongs to the orbit $\mathbf{R}_{n}$.

b) Curves with diagram in family B. If the Gauss diagram of $C$ is $\Gamma(n, n)$ $(n=2 m+1, m>0)$, then any simple arc of the circle $\gamma$ of $\Gamma(n, n)$ (i.e. any arc joining neighboring vertices) determines with its antipodal arc the boundary of a 
biangular tile of the tiling of $C$; these biangular tiles have the properties described in point 4 of the Theorem, and so $C$ belongs to the orbit $\mathbf{S}_{n}$.

c) Curves with diagram in family $\mathrm{C}$. A Gauss code of $\Gamma(4,3)$ is

$$
\Omega(4,3)=A_{0} a_{3} A_{1} a_{0} A_{2} a_{1} A_{3} a_{2}
$$

The simple arcs $A_{0} a_{3}, A_{3} a_{2}$ and $a_{0} A_{2}$ of the circle $\gamma$ of $\Gamma(4,3)$ determine the sides of a triangular tile $\Delta_{0}$ of $C$. We define in the same way $\Delta_{1}$ by means of $A_{1} a_{0}, A_{0} a_{3}$ and $a_{1} A_{3}, \Delta_{2}$ by means of $A_{2} a_{1}, A_{1} a_{0}$ and $a_{2} A_{0}$, and $\Delta_{3}$ by means of $A_{3} a_{2}, A_{2} a_{1}$ and $a_{3} A_{1} ;$ as $\Delta_{0}$ and $\Delta_{1}$ have a common side, as well as $\Delta_{1}$ and $\Delta_{2}, \Delta_{2}$ and $\Delta_{3}, \Delta_{3}$ and $\Delta_{0}$, and so these four tiles form a belt having the properties described in point 5 of the Theorem, which implies that $C$ belongs to the orbit $\mathbf{T}_{4}$. In the same way, one proves that, if the Gauss diagram $\Gamma(n, s)$ of $C$ is $\Gamma(6 m-2,4 m-1)(m>1)$ or $\Gamma(6 m+2,4 m+1)(m>0)$, then $C$ belongs to the orbit $\mathbf{T}_{n}$.

\section{DP-homogeneous plane curves}

COROLLARY: If a DP-homogeneous plane curve has at least one double point, then it belongs to one orbit (under the group of all diffeomorphisms of $\mathbb{R}^{2}$ ) of one of the three families described below:

1) The family $\mathbf{P}^{\prime}$ is the sequence of orbits $\mathbf{P}_{1}^{\prime}, \mathbf{P}_{2}^{\prime}, \mathbf{P}_{3}^{\prime}, \ldots, \mathbf{P}_{n}^{\prime}, \ldots$ where any element of $\mathbf{P}_{1}^{\prime}$ is a figure-eight curve and, if $n>1$, where any element $C$ of $\mathbf{P}_{n}^{\prime}$ is a curve (with $n$ double points) one tile of which is a curvilinear n-gon with salient vertices, each of them being also the vertex of a curvilinear 1-gon inscribed in $C$. Examples of elements of $\mathbf{P}_{1}^{\prime}, \mathbf{P}_{2}^{\prime}, \mathbf{P}_{3}^{\prime}$ and $\mathbf{P}_{4}^{\prime}$ are shown in Figure 1.

2) The family $\mathbf{P}^{\prime \prime}$ is the sequence of orbits $\mathbf{P}_{1}^{\prime \prime}, \mathbf{P}_{2}^{\prime \prime}, \mathbf{P}_{3}^{\prime \prime}, \ldots, \mathbf{P}_{n}^{\prime \prime}, \ldots$ where any element of $\mathbf{P}_{1}^{\prime \prime}$ is equivalent to a Pascal snail with inner loop and, if $n>1$, where any element of $\mathbf{P}_{n}^{\prime \prime}$ is a curve $C$ (with $n$ double points) in which a curvilinear $n$-gon $D$ with re-entrant vertices is inscribed; every vertex of $D$ is also the vertex of a curvilinear 1-gon inscribed in C. Examples of elements of $\mathbf{P}_{1}^{\prime \prime}, \mathbf{P}_{2}^{\prime \prime}, \mathbf{P}_{3}^{\prime \prime}$ and $\mathbf{P}_{4}^{\prime \prime}$ are shown in Figure 2.

3) The family $\mathbf{S}^{\prime}$ is the sequence of orbits $\mathbf{S}_{3}^{\prime}, \mathbf{S}_{5}^{\prime}, \mathbf{S}_{7}^{\prime}, \ldots, \mathbf{S}_{2 m+1}^{\prime}, \ldots$ where any element $C$ of $\mathbf{S}_{2 m+1}^{\prime}$ is a curve (with $2 m+1$ double points) one tile of which is a curvilinear $(2 m+1)$-gon $D$ with salient vertices; $D$ is separated from the unbounded tile of $C$ by $a$ chain of $2 m+1$ biangular tiles. Examples of elements of $\mathbf{S}_{3}^{\prime}, \mathbf{S}_{5}^{\prime}$ and $\mathbf{S}_{7}^{\prime}$ are shown in Figure 8.

Proof: As $\mathbb{R}^{2}$ is diffeomorphic to the complement of a point (denoted by $\infty$ ) in $S^{2}$, we may identify $\mathbb{R}^{2}$ with $S^{2} \backslash \infty$. Any DP-homogeneous plane curve $C$ may be seen as a DP-homogeneous spherical curve which, by the Theorem, belongs to one orbit of one of the families $\mathbf{P}, \mathbf{Q}, \mathbf{R}, \mathbf{S}$ and $\mathbf{T}$.

Suppose that the spherical curve $C$ belongs to $\mathbf{P}_{1} ;$ if $\infty$ is a point of a 1-gonal tile, then $C$ belongs (as a plane curve) to $\mathbf{P}_{1}^{\prime \prime}$; if not, then $C$ belongs to $\mathbf{P}_{1}^{\prime}$.

If $C$ belongs to $\mathbf{P}_{n}$ with $n>1$, then $\infty$ cannot be a point of a 1-gonal tile, which implies that $C$ belongs to $\mathbf{P}_{n}^{\prime}$ or $\mathbf{P}_{n}^{\prime \prime}$.

If $C$ belongs to $S_{n}$, then $\infty$ cannot be a point of a 2-gonal tile, which implies that $C$ belongs to $\mathbf{S}_{n}^{\prime}$. 
If $C$ belongs to $\mathbf{Q}_{n}, \mathbf{R}_{n}$ or $\mathbf{T}_{n}$, then every position of $\infty$ leads to a contradiction, which proves that, in the classification of the orbits of DP-homogeneous plane curves, there is no familiy other than $\mathbf{P}^{\prime}, \mathbf{P}^{\prime \prime}$ and $\mathbf{S}^{\prime}$.

\section{DP-homogeneity in the real projective plane}

Family P'
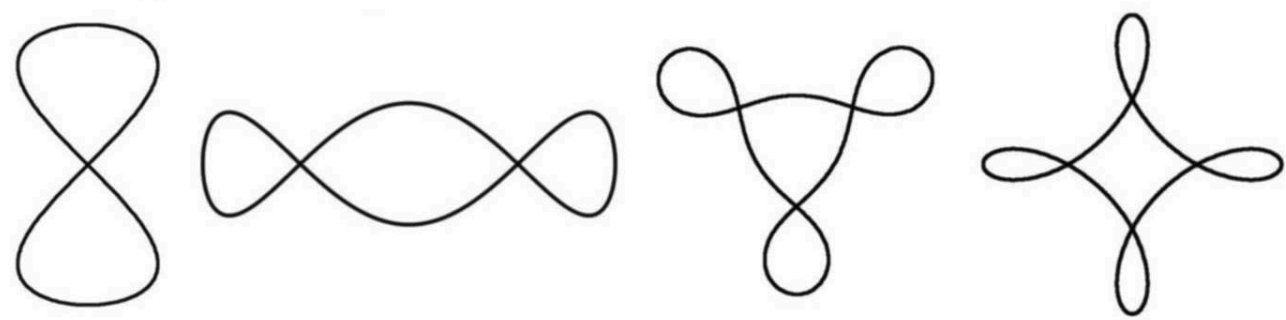

etc.

Family P"
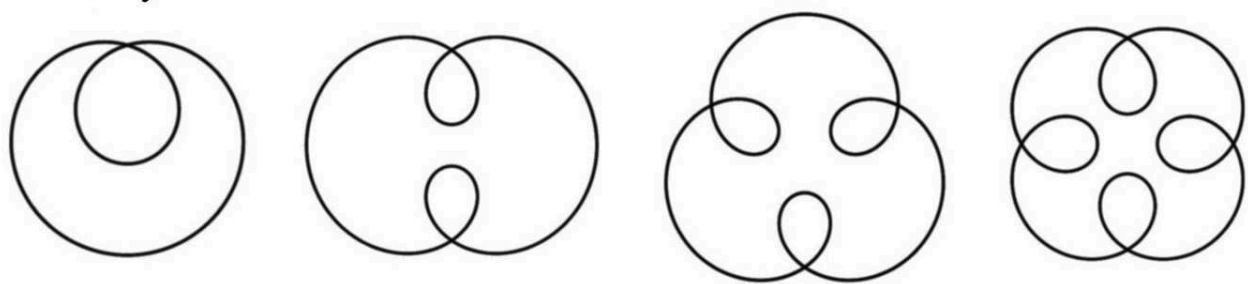

etc.

Family $\mathbf{S}^{\prime}$
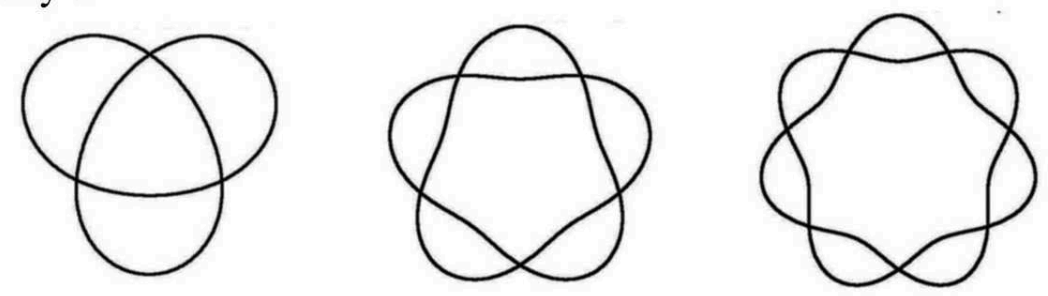

etc.

Family $\mathbf{S}$
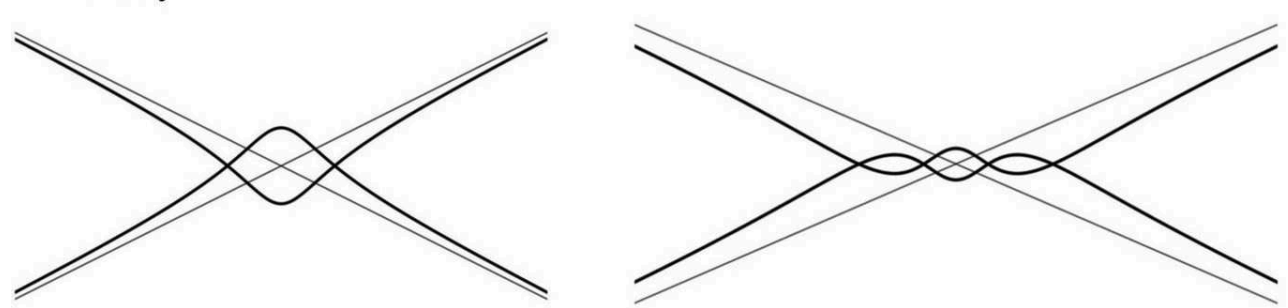

etc.

FIGURE 13: Representatives of some elements of the four families of orbits of nullhomotopic DP-homogeneous curves in the real projective plane.

CONJECTURE: Let $C$ be a DP-homogeneous curve of $P_{2}(\mathbb{R})$ with at least one double point.

1) If $C$ is null-homotopic, then it belongs to one orbit (under the group of all diffeomorphisms of $P_{2}(\mathbb{R})$ ) of one of four infinite families: 
a) The family $\mathbf{P}^{\prime}$ is the sequence of orbits $\mathbf{P}_{n}^{\prime}\left(n \in \mathbb{N}_{0}\right)$, whose representatives are curves with $n$ double points sketched, for $n<5$, in the first row of Fig. 13.

b) The family $\mathbf{P}^{\prime \prime}$ is the sequence of orbits $\mathbf{P}_{n}^{\prime \prime}\left(n \in \mathbb{N}_{0}\right)$, whose representatives are curves with $n$ double points sketched, for $n<5$, in the second row of Fig. 13.

c) The family $\mathbf{S}^{\prime}$ is the sequence of orbits $\mathbf{S}_{n}^{\prime}\left(n=2 m+1, m \in \mathbb{N}_{0}\right)$, some representatives of which are sketched, for $n<8$, in the third row of Fig. 13.

d) The family $\mathbf{S}^{\prime \prime}$ is the sequence of orbits $\mathbf{S}_{n}^{\prime \prime}\left(n=2 m, m \in \mathbb{N}_{0}\right)$, some representatives of which are sketched, for $n<5$, in the last row of Fig. 13.

2) If $C$ is not null-homotopic, then it belongs to one orbit (under the group of all diffeomorphisms of $P_{2}(\mathbb{R})$ ) of one of three infinite families:

a) The family $\mathbf{Q}^{\prime}$ is the sequence of orbits $\mathbf{Q}_{n}^{\prime}(n=2 m+1, m \in \mathbb{N})$, whose representatives are sketched, for $n<6$, in the upper part of Fig. 14 .

b) The family $\mathbf{R}^{\prime}$ is the sequence of orbits $\mathbf{R}_{n}^{\prime}(n=4 m+2, m \in \mathbb{N})$, whose representatives are sketched, for $n<7$, in the lower part of Fig. 14 .

c) The family $\mathbf{T}^{\prime}$ is the sequence of orbits $\mathbf{T}_{n}^{\prime}(n=6 m-1$ or $n=6 m+1$, $m \in \mathbb{N}_{0}$ ) whose representatives are sketched, for $n<8$, in Fig. 15.

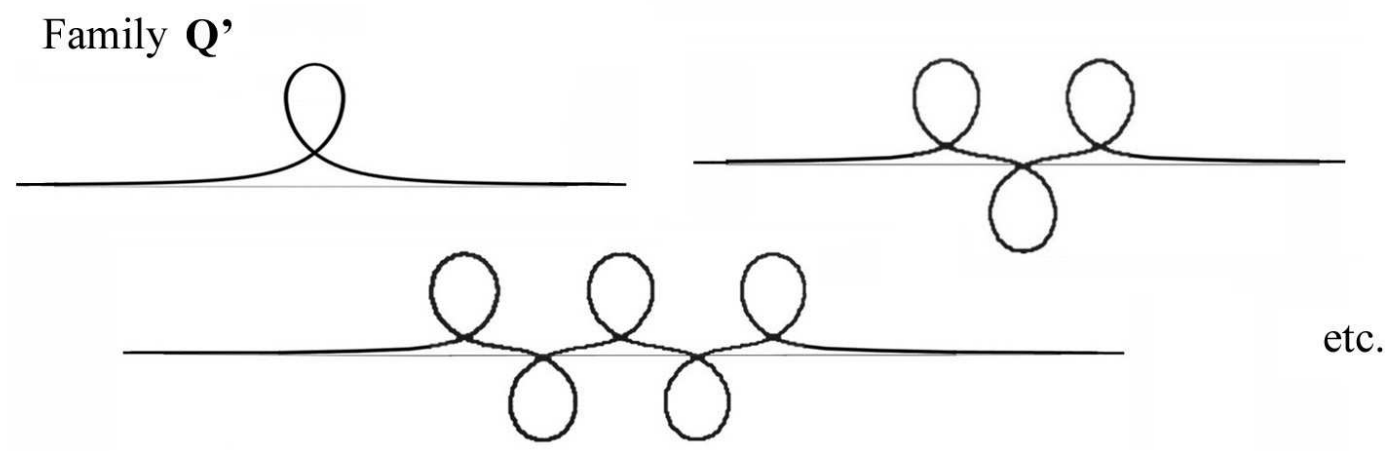

\section{Family R'}

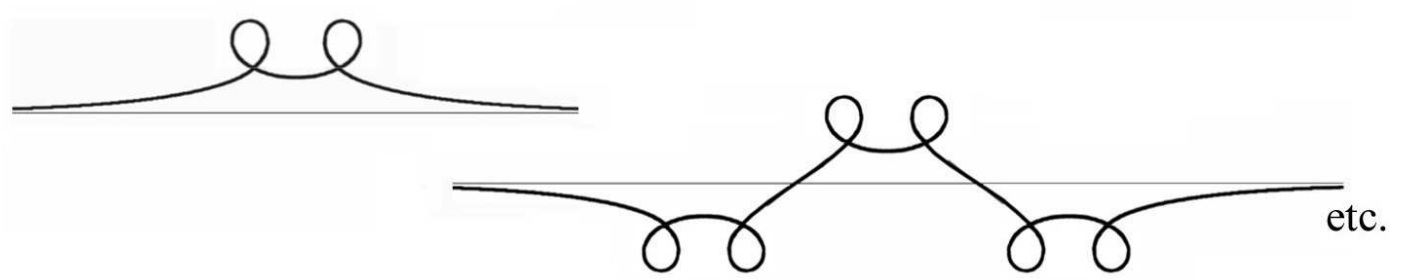

FIGURE 14: Representatives of some elements of the families of orbits $\mathbf{Q}^{\prime}$ and $\mathbf{R}^{\prime}$ of non null-homotopic DP-homogeneous curves in the real projective plane. 


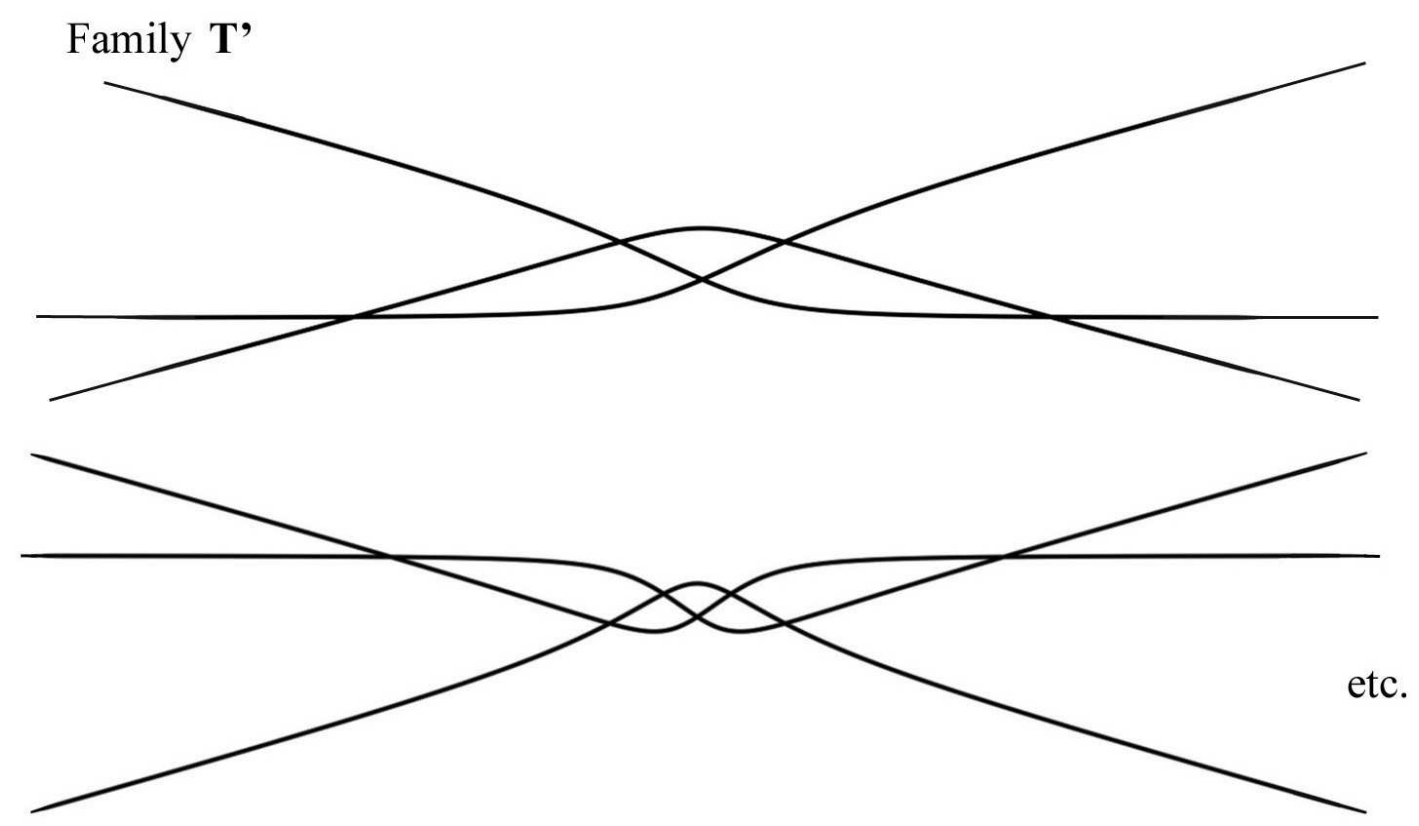

FIGURE 15: Representatives of the orbits $\mathbf{T}_{5}^{\prime}$ and $\mathbf{T}_{7}^{\prime}$ of non null-homotopic DP-homogeneous curves in the real projective plane.

Acknowledgments : I should like to express my grateful thanks to J.-P. Doignon and J.-B. Zuber who encouraged me to write and publish this work.

\section{References}

[Ga] C.F. GAUSS, Zur Geometria Situs, Werke, vol. 8, p. 271-286.

[LM] L. LOVASZ, M.L. MARX A forbidden substructure characterization of Gauss codes, Acta Sci. Math. Soc. 38 (1976), p. 115-119.

Emeritus Professor, Department of Mathematics,

Vrije Universiteit Brussel,

Pleinlaan 2, B1050 Brussels, Belgium

email: guy.valette@vub.ac.be 